$10.2478 / \mathrm{gb}-2020-0001$

sciendo

\title{
Heiter bis wolkig. Zum 50. Jubiläum des Hermannstädter Lehrstuhls für Germanistik
}

\section{Doris SAVA}

Doz. Dr.; Lucian-Blaga-Universität Sibiu/Hermannstadt;

E-Mail: doris.sava@ulbsibiu.ro

\begin{abstract}
German Studies in Hermannstadt (Sibiu) have always put an increased focus on preserving continuity and consistency in teaching and research. The international anniversary conference (October 2019) that marked 50 years since the establishment of the Chair of German Studies in Hermannstadt (Sibiu) offered ample opportunity for taking a retrospective look in order to reflect on continuities and ruptures. Thus, in the article are mentioned some of the challenges that the chair had to face in its recent history.
\end{abstract}

Keywords: anniversary of the establishment, Chair of German Studies in Hermannstadt (Sibiu), continuity, change

Ein rundes Jubiläum ist freilich zunächst ein Anlass zum Feiern. Im Herbst 2019 galt es doppelt zu feiern: 50 Jahre Germanistik in Hermannstadt (Sibiu) ${ }^{1}$ und den 60 . Geburtstag von Maria Sass, langjähriger Leiterin des hiesigen Germanistiklehrstuhls (2004-2015). Dem Fachgebiet ist gutes Gedeihen zu wünschen, der Jubilarin und den Mitgliedern des Lehrstuhls wissenschaftliche Neugier und Gesundheit, verbunden mit der Ermutigung, zuversichtlich an die Zukunft ihres Faches zu denken. Denn: Es gibt noch viel zu tun.

Der Wahrung von Kontinuität und Konstanz in der Lehre und Forschung hat die Hermannstädter Germanistik stets erhöhtes

${ }^{1}$ Fort an wird ausschliesslich der historische, deutsche Name der Stadtverwendet. 
Augenmerk geschenkt. Die zum 50. Gründungstag des Hermannstädter Lehrstuhls für Germanistik veranstaltete internationale Jubiläumstagung Literatur und Sprache im südosteuropäischen Raum (24.-26. Oktober 2019) bot ausreichend Gelegenheit, Rückschau zu halten ${ }^{2}$, über Kontinuitäten und Brüche zu reflektieren und auch einige Herausforderungen zu benennen, denen sich der Lehrstuhl in diesen bewegten Zeiten finanzieller Engpässe, des Konkurrenzdrucks und der Anpassung an das von reinem Effizienzdenken gesteuerte Agieren bildungspolitischer Entscheidungsträger stellen musste. Hierzu gehört auch der willkürliche Zusammenschluss ${ }^{3}$ der beiden Lehrstühle für Germanistik und Anglistik zum Departement für anglo-amerikanische und germanistische Studien (2011). In der hier gebotenen Kürze seien im Folgenden einige Stationen in der jüngsten Geschichte unseres Lehrstuhls erwähnt.

Zum 50. Gründungstag des Hermannstädter Lehrstuhls für Germanistik wurden in den Begrüßungsansprachen nicht nur Kontinuitäten und Brüche benannt und reflektiert, sondern auch das Profil dieser Germanistik betont. Was macht also das Besondere der Germanistik vor Ort aus? Nun, es sind zunächst ihre Träger selbst gewesen, die Hochschullehrer, verkörperten sie doch Tugenden wie Fleiß, Zuverlässigkeit oder Gemeinschaftssinn - kurzum: Pflichtbewusstsein. Zu den menschlichen Qualitäten der Lehrkräfte gehören zweifelsohne eine gewisse Diszipliniertheit, womit Ernsthaftigkeit und Gewissenhaftigkeit gleichfalls gemeint sind, sowie ein Gespür für das Umsetzbare,

2 Siehe dazu auch folgende Berichte: Ungar, Beatrice: „Eine zuversichtliche Gemeinschaft": 50. Gründungsjubiläum der Germanistik in Hermannstadt. In: Hermannstädter Zeitung, 25.10.2019; unter https:// www.hermannstaedter.ro/2019/10/nachrichten-315/; 11.12.2019 und Fischer, Markus: 50 Jahre Germanistik in Hermannstadt. Internationale Jubiläumstagung an der Lucian-Blaga-Universität. In: Allgemeine Deutsche Zeitung für Rumänien, 4.11.2019; unter https://adz.ro/artikel/artikel/50-jahre-germanistik-in-hermannstadt; 11.12.2019.

3 Nach den Regelungen des neuen Bildungsgesetzes (2011). 
die schwierigen administrativen und bildungspolitischen Hürden umgehend. Auch die Tradition weiterhin zu wahren, das bisher Erreichte in der Lehre und Forschung im Umfeld neuer Aufgaben und Herausforderungen fortzusetzen, ist das Ergebnis gemeinsamer Anstrengungen und wurde daher nicht in Eigenregie, jedoch mit Ausdauer betrieben. So waren die Leiter der Hermannstädter Germanistik oder die hier Wirkenden zugleich Anreger und Mitgestalter zahlreicher gemeinschaftlicher und oft in Kooperation verwirklichten Forschungsvorhaben, literarischer und wissenschaftlicher Veranstaltungen oder Publikationen. Dies alles und noch einiges mehr begründete die Standhaftigkeit der Hermannstädter, denen von der inländischen Fachgemeinschaft auch eine gewisse Selbstgefälligkeit nachgesagt wird, die sich am „engen siebenbürgischen Horizont“" verausgaben würde... Tatsache ist, dass sich die Hermannstädter für eine gemeinsame Sache einzusetzen gewusst haben, gemeinsam an nachhaltigen Zukunftskonzepten gearbeitet und gemeinsam Auswege aus den Wirren gefunden haben. Beachtung verdient auch ihr Engagement, sich dort einzubringen, wo es galt, fachliches Wissen und Können gezielt anzuwenden und auch geeignete Fachkollegen und Mitarbeiter hierfür anzuwerben. Angesichts des weiter unten geschilderten Sachverhalts und einer langjährigen akuten lähmenden Trägheit seitens der Institutsverwaltung sicherlich kein leichtes Unterfangen!

Betrachter aus der Nähe würden daher unschwer die Kontinuität der Anstrengungen ignorieren können, die der Hermannstädter Lehrstuhl für Germanistik über Jahre auf sich genommen hat, um den Lehr- und Forschungsbetrieb aufrecht zu erhalten. Nicht ruhen und nicht rasten wollte und sollte die Hermannstädter Germanistik, die sich durch die kontinuierliche Ausrichtung auf das Lokalspezifische, auf das vor Ort Auffindbare und dessen wissenschaftlich Nutzung nach dem Umbruch 1990 in eine Nische gerettet hatte.

In den guten Zeiten, den ,goldenen 1970er-Jahren“, regte Hermannstadt, und hier nicht nur der Lehrstuhl, sondern auch 
eine andere, der Forschung zugewandten Einrichtung, das Institut für Geisteswissenschaften und Außenstelle der Rumänischen Akademie, Forschungsvorhaben literaturgeschichtlicher Prägung in Zusammenarbeit mit anderen Lehrstühlen des Landes an. Joachim Wittstock (2010: 16) formulierte dies so ${ }^{4}$ :

Germanistische Forschung wird begünstigt, wenn sie, außer auf Themen allgemeiner Verbindlichkeit, sich auf Sachverhalte regionaler Zuordnung einstellen kann, auf Gegebenheiten außerhalb des binnendeutschen Sprachraums. In Siebenbürgen hat man germanistische Forschungsfelder spezifischen Charakters abstecken und bearbeiten können [...]. Die Gunst des Standorts Hermannstadt/Sibiu - siebenbürgisch-sächsischer Dialekt im Umfeld, urbanes und suburbanes Deutsch im Prozess der Angleichung, Zugang zum deutschen Schriftum der Region und der benachbarten Provinzen, zudem pädagogisch-schulische Voraussetzungen für ergebnisreichen Sprachunterricht - diese Vorzugsstellung hat die wissenschaftliche Auseinandersetzung um Autoren und Werke rumäniendeutscher Literatur gefördert. Kein Wunder also, dass die literaturgeschichtliche Untersuchung des Erbes und der Gegenwartsproduktion im Blickfeld der Fachkräfte war. Befürworter, Träger und Planer hiesiger Hochschulgermanistik ließen, wie angedeutet, literarhistorische Forschung als Wunschziel nicht aus den Augen.

In den Eröffnungsansprachen wurden folglich nicht nur das Wirken der Lehrstuhlmitglieder gewürdigt, sondern auch die Bemühungen aller Mitarbeiter von nah und fern, die seit der Lehrstuhlgründung am 15. Juni 1969 an der Fakultät für Philologie und Geschichte, eine Außenstelle der Klausenburger BabeșBolyai-Universität an der auch die Fachbereiche Anglistik und Romanistik angesiedelt waren, den Hermannstädter Lehrstuhl

${ }^{4}$ Im Zusammenhang mit dem Großprojekt Geschichte der deutschen Literatur Siebenbürgens, wobei die Koordination der Zeitspanne 18491944 Hermannstadt anvertraut wurde. Vgl. Wittstock, Joachim: Aus dem Bereich von „Litteris et Artis“. Literarhistorische Erörterungen im Rahmen der Hermannstädter Hochschulgermanistik. In: Germanistische Beiträge 26/2010, S. 15-30. 
vertreten haben. ${ }^{5}$ Im Jahr 1976 erfolgte die Loslösung von der Klausenburger Universität und die Umwandlung in ein Hochschulinstitut. Visionäre Stimmen aus dem Lehrbetrieb und auch aus der Verwaltung sahen in der angestrebten Selbstständigkeit den Grund für die spätere Auflösung dieser Einrichtung, die 1984 infolge der Sparmaßnahmen auch erfolgte. Viele Mitarbeiter wanderten aus. Nach der Wende 1990 wurden die noch im Lande Verbliebenen ${ }^{6}$ an die wiedergegründeten Lehrstühle gerufen, womit nicht nur ein enormer Verwaltungsaufwand verbunden war, sondern auch die Anwerbung neuer Mitglieder, darunter ehemalige Studierende.

Der Lehrstuhl für Germanistik in Hermannstadt hat seine Aufgabe stets zu pflegen verstanden ${ }^{7}$. Entgegen allen Widrigkeiten war und ist in Anlehnung an die humboldtsche Einheit von Lehre und Forschung die Förderung des wissenschaftlichen Austausches und des Nachwuchses ein wichtiges Anliegen unseres Lehrstuhls. Diesem Ziel dienen die durchgeführten

${ }^{5}$ Im Jahre 1984 erfolgte die willkürliche Auflösung der Fakultät. Nach dem politischen Umbruch 1990 wurde durch einen Regierungsbeschluss die Philologie und die Germanistik an der Universität in Hermannstadt, die seit dem 12. Mai 1995 den Namen des bekannten Dichters und Philosophen Lucian Blaga (1895-1961) trägt, neu gegründet.

6 Die Gründung der ersten Lehrstühle für Germanistik an rumänischen Universitäten (Bukarest 1905, Iasi 1905, Cluj 1919) diente der Ausbildung von Deutschlehrern bzw. der Verbreitung der deutschen Kultur. Die Entwicklung der Germanistik in Rumänien wurde vor und nach der politischen Wende (1989/1990) durch die deutsche Minderheit geprägt. Lehrende an deutschen Schulen in Rumänien waren bis zur Wende fast ausschließlich Angehörige der deutschen Minderheit. Nach der massiven Rückwanderung der Rumäniendeutschen in den 1990erJahren ist die Zahl der Deutschstämmigen in Rumänien drastisch zurückgegangen.

7 Vgl. hierzu Konnerth, Gerhard: Der Hermannstädter Lehrstuhl für Germanistik. Ausblick und Rückschau. In: Germanistische Beiträge 13/14, 2000, S. 18-31. 
Forschungsprojekte, Tagungen mit ihren Foren für Jungwissenschaftler und die vom Lehrstuhl betreuten Publikationen.

In den Grußworten der Universitätsvertreter und Fakultätsleitung ist daher auch die Bedeutung der Hermannstädter Germanistik für die Fakultät insgesamt hervorgehoben worden, die sich in der Kontinuität ihrer Veranstaltungen und Kooperationen wie auch in der Herausgabe der Lehrstuhlpublikation Germanistische Beiträge äußert. Es ist schon beachtlich, wenn es einem kleinen Lehrstuhl gelingt, in einem Jahr zwei Publikationen herauszugeben, die sich im Umfang auf 830 Seiten berufen. Zwei Neuerscheinungen, beide Festschriften ${ }^{8}$ für Hermannstädter Germanisten, die auch zu den Teilnehmern der Jubiläumstagung zählten, sind pünktlich zur akademischen Würdigungsfeier im Berliner Verlag Peter Lang erschienen. Bei den Geehrten handelt es sich um Maria Sass, der Hermannstädter Germanistik Kopf und Seele zugleich, und um den Gelehrten und Schriftsteller Joachim Wittstock. ${ }^{10}$ Vieles von

8 Literaturgeschichte und Interkulturalität. Festschrift für Maria Sass (532 S.) hrsg. von Doris Sava und Stefan Sienerth und Rumäniendeutsche Seinszusammenhänge und weitläufigere Bezüge. Literarische Kommunikation in der deutschsprachigen Literatur Rumäniens - das Fallbeispiel Joachim Wittstock (298 S.) hrsg. von Maria Sass, Olivia Spiridon und Stefan Sienerth.

9 Maria Sass hat das Studium der Germanistik und Rumänistik an der Fakultät für Philologie und Geschichte in Hermannstadt 1983 als Jahresbeste beendet. Ihren wissenschaftlichen Werdegang begann sie als Assistentin von Prof. Dr. Horst Schuller Anfang der 1990er-Jahre. 1998 promovierte sie bei ihm über die Beziehungen von George Coşbuc (1866-1918) zur deutschen Literatur. Nach seiner Emeritierung 2002 ermutigte Horst Schuller Maria Sass, die Leitung des Lehrstuhls zu übernehmen. Maria Sass war von 2004 bis 2011 Lehrstuhlinhaberin und von 2011 bis 2015 Leiterin des Departements für anglo-amerikanische und germanistische Studien.

${ }^{10}$ Dr.h.c. Joachim Wittstock ist Dichter, Prosaautor, Essayist, Literaturhistoriker und Übersetzer. Zu seinem 60. Geburtstag 1999 wurde ihm die Ehrendoktorwürde der Lucian-Blaga-Universität verliehen. Zu 
dem, was zum Wohl der Hermannstädter Germanistik in die Wege geleitet wurde, wäre ohne die guten Verbindungen und menschlichen Beziehungen der Lehrstuhlleiter nach der Wende, Gerhard Konnerth ${ }^{11}$, Horst Schuller ${ }^{12}$ Sunhild Galter und Maria Sass, überhaupt nicht oder nicht sofort verwirklicht worden.

Wie sehr die deutsche Philologie geschätzt wird, zeigt sich auch in der Anvertrauung des Forschungszentrums ${ }^{13}$ der Philologischen Fakultät nach der Wiedergründung 2017 einer Germanistin wie auch in der starken Vertretung der Germanistik

seinem literarischen Schaffen vgl. den Sonderband der Germanistischen Beiträge (Band 25/2009) von Maria Sass und Sunhild Galter betreut: Schreiben ist zweifellos ein Ziegelrücken. Studien und Aufsätze zu Joachim Wittstocks Werk (S. 148-170)/Joachim Wittstock: Bibliographie 1999-2009 (S. 173-194). Joachim Wittstock ist (Mit-)Verfasser zahlreicher Studien und kollektiv erarbeiteten Darstellungen zum deutschsprachigen Schrifttum Siebenbürgens, des Banats und der Bukowina. Er betreute als Korrektor, Redaktionssekretär oder Chefredakteur(38/1995-41/1998) die einzige deutschsprachige Publikation der Rumänischen Akademie Forschungen zur Volks- und Landeskunde (1959 gegründet).

${ }^{11}$ Bleibende Verdienste für den Lehrstuhl und die Hermannstädter Philologie sind mit dem vielseitigen Wirken von Gerhard Konnerth bis zu seiner Emeritierung 2006 verbunden. In der Verwaltung tätig als Dekan (1976-1984) und Prodekan (1973-1976, 1990-1995, 20002004), Lehrstuhlinhaber (1990-2000).

${ }^{12}$ Horst Schuller kam 1990 an den neu gegründeten Lehrstuhl für Germanistik. Bis zu seiner krankheitsbedingten Emeritierung 2002 war er maßgeblich an der inhaltlichen Neuorientierung und Umgestaltung der Hermannstädter Germanistik beteiligt, im Zeitraum 2000-2002 auch als Leiter des Lehrstuhls. Nach seiner Ausreise 2003 blieb er als beratender Professor und Doktorvater (fast 30 angeleitete Arbeiten) diesem Lehrstuhl verbunden. Seine Nachfolgerin war Dr. Sunhild Galter, die zwischen 2002 und 2004 den Lehrstuhl leitete.

${ }^{13}$ Zentrum für linguistische, literarische und kulturelle Forschung (ZLLKF); rum. Centrul de studii lingvistice, literare și culturale (CSLLC). 
im Leitungsrat ${ }^{14}$ des Departements für anglo-amerikanische und germanistische Studien.

Durch internationale Kooperationen, Projektinitiierungen, Tagungen und die Herausgabe von Publikationen gelang der Hermannstädter Germanistik eine dauerhafte Verortung im Inland und Ausland. Die langjährige Tradition und der hohe Stellenwert, den die Pflege und die Vermittlung der deutschen Literatur, Sprache und Kultur am Germanistik-Lehrstuhl der Lucian-Blaga-Universität in Sibiu/Hermannstadt einnimmt, haben die Zusammenarbeit mit unterschiedlichen Institutionen und Einrichtungen gefördert und die regelmäßige Durchführung von gemeinsamen interdisziplinären Forschungsprojekten oder von wissenschaftlichen Tagungen ermöglicht. Zu den traditionellen und aktivsten Kooperationspartnern der Hermannstädter Germanistik gehören ab 2005 das Institut für deutsche Kultur und Geschichte Südosteuropas an der Ludwig-MaximiliansUniversität (IKGS) in München, die Donauschwäbische Kulturstiftung des Landes Baden-Württemberg oder die EuropaUniversität Flensburg.

Im Hinblick auf die wissenschaftliche Zusammenarbeit gilt es daher auf einige erfolgreiche, thematisch miteinander verknüpfte Projektvorhaben zu verweisen, deren Ergebnisse auch in Buchform aufliegen. Zusammen mit der rumänischen Philologie wurde 2018 ein Gemeinschaftsprojekt gestartet, das dem literarischen Übersetzen gewidmet war. ${ }^{15} \mathrm{Zu}$ den vom IKGS

${ }^{14}$ Nach den Departementswahlen im November 2019 ist die Germanistik durch Maria Sass, Sunhild Galter und Doris Sava vertreten. Die Leitung des Departements übernahm 2019 erneut ein Anglist, Dr. Ovidiu Matiu (seit 2015 im Amt).

${ }^{15}$ Vgl. Sass, Maria/Baghiu, Ștefan/Pojoga, Vlad (Hgg.): The Culture of Translation in Romania/Übersetzungskultur und Literaturübersetzen in Rumänien. Berlin, 2018; ferner auch Baghiu, Ștefan/Pojoga, Vlad/ Sass, Maria (Hgg.): Ruralism and Literature in Romania. Berlin 2019. Für den zweisprachigen Band Übersetzungskultur und Literaturübersetzen in Rumänien (2018) wurde Maria Sass mit dem Sonderpreis 
mitgetragenen Jahrestagungen der Hermannstädter Germanistik, die Prof. Dr. Dr. h.c. Stefan Sienerth, zwischen 2005 und 2013 Direktor des IKGS, vollumfänglich unterstützt hat, gesellen sich auch weitere Tagungen, die thematisch auf die Forschungsfelder der hiesigen Germanistik und des erwähnten Instituts ausgerichtet waren ${ }^{16}$ oder auf das Werk und Wirken von namhaften zeitgenössischen rumäniendeutschen und rumänischen Autoren und deren Übersetzer als Vermittler von Literatur und Kultur fokussieren. ${ }^{17}$ Die von Maria Sass einberufenen Jahrestagungen beleuchten Wirkungszusammenhänge von Sprache und Literatur, während die Tagungen germanistischer Nachwuchswissenschaftler (ab 2010) der Interkulturalität und der Bestandsaufnahme rumänisch-deutscher Kulturbeziehungen, die Hermannstadt und Siebenbürgen prägten, gewidmet waren ${ }^{18}$. Die traditionelle Jahrestagung (kontinuierlich ab 2006) der Germanistik ist die langlebigste Veranstaltung der

der Rumänischen Gesellschaft für Allgemeine und Vergleichende Literaturwissenschaft (rum. Asociația de Literatură Generală și Comparată din România ALGCR) anlässlich der 16. Jahrestagung Das deutsche Kulturmodell in der rumänischen Kultur (Hermannstadt, 10. - 13. Juli 2019) ausgezeichnet.

${ }^{16}$ Aus der langjährigen Kooperation des IKGS mit wissenschaftlichen Instituten und Universitäten aus Südosteuropa gingen internationale Symposien und Tagungen hervor, die der deutschsprachigen Literatur Südosteuropas gewidmet waren. Vgl. hierzu folgende Tagungsbände: Miladinović Zalaznik, Mira/Sass, Maria/Sienerth, Stefan (Hgg.): LiterarischeZentrenbildungin Ostmittel-und Südosteuropa: Hermannstadt/ Sibiu, Laibach/Ljubljana und weitere Fallbeispiele. München 2010 und Galter,Sunhild/Sass, Maria/Tichy,Ellen(Hgg.): Wechselwirkungen im deutsch-rumänischen Kulturfeld. Beiträge zu Sprach- und Literaturkontakten aus interkultureller Perspektive. Frankfurt/Main 2015.

${ }^{17}$ Vgl. den Tagungsband: Sass, Maria/Sava, Doris/Sienerth, Stefan (Hgg.): Schriftsteller versus Übersetzer. Begegnungen im deutsch-rumänischen Kulturfeld. Frankfurt/Main 2013.

${ }^{18}$ Zur Dokumentation der Tagungsarbeiten vgl. Sass, Maria: Germanistische Beiträge Monographien: Beiträge germanistischer Nachwuchskräfte, Bd. I-IV. Sibiu 2011-2014. 
Hermannstädter Philologie, die von Lehrenden und Nachwuchswissenschaftlern gleichermaßen besucht wird.

Ab 2011 übernimmt die Donauschwäbische Kulturstiftung des Landes Baden-Württemberg eine aktive Rolle in der finanziellen Förderung des Lehrstuhls. Seit 2012 hat Maria Sass in Zusammenarbeit mitdieser Kulturstiftung die Sommerakademie in Hermannstadt veranstaltet und angeleitet ${ }^{19}$. Das jährliche Zusammentreffen vereinigt Studierende sowie Dozenten aus fünf Ländern, die Interesse an der deutschen Sprache und Kultur im internationalen Kontext haben: Siedlungsgeschichte, Landschaft und Städte Siebenbürgens, rumäniendeutsche Literatur und deutsche Kultur in Siebenbürgen, die gegenwärtige Situation der deutschen Minderheit. Zu den Stärken des Programms gehören Lesungen und Gespräche mit den rumäniendeutschen Schriftstellern Eginald Schlattner, Joachim Wittstock und Walther Gottfried Seidner sowie Exkursionen (Sighișoara, Mediaş, Braşov, Cisnădioara, Biertan, Cisnădie, Prejmer, Bran, Albota oder Ocna Sibiului).

$\mathrm{Zu}$ den wesentlichenAufgaben der hiesigen Germanistik gehört - knapp erfasst - die Wahrung einer Kulturtradition: die Erforschung des siebenbürgischen Schrifttums, dessen literaturgeschichtliche Untersuchung sowie die Pflege und Vermittlung der rumäniendeutschen Literatur und Sprache. Zu den bevorzugten Forschungsgebieten der Hermannstädter gehören daher vornehmlich die rumäniendeutsche (Gegenwarts-)Literatur (Erwin und Joachim Wittstock, Erwin Neustädter, Walther

${ }^{19}$ Vgl. hierzu Sass, Maria: Deutsche Sprache, Literatur und Kultur in Rumänien - Bilanz und Perspektiven. In: Christ, Eugen/Peter Dines/ Stefan Jeuk (Hgg.): 25 Jahre Donauschwäbische Kulturstiftung des Landes Baden-Württenberg (1988-2013). Bruchsal 2013, S. 43-52, 2013 und Sass, Maria: Tradition heißt nicht, die Asche aufheben, sondern die Flamme weiterreichen: Die Sommerakademie „Siebenbürgen“ und ihr Beitrag zur Förderung der deutschen Sprache, Kultur und des Austausches in Südosteuropa. In: Germanistische Beiträge 33/2013, S. 225-244. 
Gottfried Seidner, Carmen E. Puchianu, Andreas Birkner, Paul Schuster, Eginald Schlattner, Ursula Ackrill u.a.), deren Rezeptionsgeschichte und Übersetzung, Wechselwirkungen im Bereich der Interkulturalität, der Literaturvermittlung und des deutschrumänischen Kulturaustausches, Reflexionen zum Stellenwert deutschsprachiger Regionalliteraturen (Banat, Bukowina und Siebenbürgen), die Beschäftigung mit Fragen der Inter-, Transund Multikulturalität, wobei aus interdisziplinärer, grenzüberschreitender Perspektive aktuelle und spezifische Forschungsfragen und Positionen aufgegriffen wurden, während im linguistischen Bereich die Problematik des Sprachkontaktes und derFach-/Berufssprachen, Überlegungen zur Übersetzungspraxis dominieren oder die Erforschung früherer Sprachstufen des Deutschen in dieser Region im Fokus stand. Historische Wandelprozesse, Dialektforschung, die Erforschung aktueller Erscheinungsformen von Sprache aus kontrastiver und variationslinguistischer Perspektive gehören ebenfalls zum Profil dieser Germanistik.

Internationale Anerkennung wurde dem Rumäniendeutschen dank der stark erweiterten Neuauflage (2016) des Variantenwörterbuchs des Deutschen (VWB) ${ }^{20}$ zuteil, die 162 standardsprachliche Lemmata aus den Viertelzentren ${ }^{21}$ Namibia, den mexikanischen Mennonitensiedlungen und Rumänien verzeichnet. Als Varietät des Deutschen weist das Rumäniendeutsche eigene Varianten (Rumänismen) auf. Es handelt sich um

${ }^{20}$ Vgl. Ammon, Ulrich/Bickel, Hans/Lenz Alexandra N. (Hgg.): Variantenwörterbuch des Deutschen. Die Standardsprache in Österreich, der Schweiz, Deutschland, Liechtenstein, Luxemburg, Ostbelgien und Südtirol sowie Rumänien, Namibia und Mennonitensiedlungen. Berlin 2016.

${ }^{21}$ Die Viertelzentren sind historisch unter ganz verschiedenen Bedingungen entstanden: infolge einer vor mehr als 800 Jahren erfolgten Ansiedlung (Rumänien), als Folge des neuzeitlichen Kolonialismus (Namibia) und als Ergebnis von Glaubensverfolgung (Übersee). Vgl. auch Kap. 4 im einleitenden Teil des VWB (2016: XXXIX-LXIV). 
typische Lexeme, Ergebnisse eines multikulturellen und mehrsprachigen Umfeldes und des Sprachkontaktes (z.B. rumänische Transferenzen, Lehnübersetzungen und hybride Wortformen). Z.B. Allgemeinschule, Aufboden, Bierfabrik, Bizikel, Bokantsch, Hanklich, Märzchen, Muskelfieber, Klettiten, Kontrollarbeit, Kulturheim, Lektionsplan, Mikrobus, Otata, Palukes, Tokane, Zuika u.a. $)^{22}$. Das Nachschlagewerk dokumentiert den Gebrauch von insgesamt 79 standardsprachlichen Rumänismen, die pressesprachlichen Modelltexten entnommen wurden und die verschiedene Themenbereiche wie Verwaltung, Schulwesen, Wirtschaft, Kochkunst, Geselligkeit, Brauchtum abdecken.

Die Anerkennung der in Rumänien gebräuchlichen ,eigenständige[n] Varietät des Deutschen mit standardsprachlicher Geltung" (Lăzărescu 2013: 370) ) $^{23}$ ist im Inland der Hermannstädter Germanistin Johanna Bottesch ${ }^{24}$, der in Iași wirkenden Namenforscherin Adina-Lucia Nistor ${ }^{25}$ und dem Bukarester Linguisten Ioan Lăzărescu ${ }^{26}$ zu verdanken, im Ausland war und

${ }^{22}$ Aus synchroner Sicht ist das in Siebenbürgen und in Rumänien, heute nicht mehr ausschließlich von den Mundartsprechern gesprochene (Hoch)Deutsch, das Rumäniendeutsche, eine überrregionale Sprachvarietät des Deutschen. Gegenwärtig wird diese Standardvarietät vom Rumänischen und Ungarischen stark beeinflusst.

${ }^{23}$ Vgl. Lăzărescu, Ioan: Rumäniendeutsch - eine eigenständige, jedoch besondere Varietät der deutschen Sprache. In: Schneider-Wiejowski, Karina/Birte Kellermeier-Rehbein/Jakob Haselhuber (Hgg.): Vielfalt, Variation und Stellung der deutschen Sprache. Berlin 2013, S. 369-389.

${ }^{24}$ Vgl. Bottesch, Johanna: Rumänien. In: Eichinger, Ludwig M./Albrecht Plewnia/Claudia Maria Riehl (Hgg.): Handbuch der deutschen Sprachminderheiten in Mittel- und Osteuropa. Tübingen 2008, S. 329-392.

${ }^{25}$ Vgl. hierzu ihre Dissertation Rumänisch-deutsche/siebenbürgisch-sächsische Sprachinterferenzen im Südwesten Siebenbürgens. Iaşi 2001.

${ }^{26}$ Im Variantenwörterbuch des Deutschen war Ioan Lăzărescu von der Universität Bukarest der verantwortliche Experte für das Viertelzentrum Rumänien. Das Standardwerk bietet neue Einsichten in die Varietätenvielfalt des Deutschen und verdeutlicht, dass die im Nachschlagewerk registrierten Auffälligkeiten nicht als Abweichungen vom Standard zu interpretieren sind, sondern als eigenes Gepräge des Deutschen außer- 
ist es - seit Jahren schon - Hermann Scheuringer, der diese Varietät, das darf mal wohl ruhig sagen, populär gemacht und dafür überhaupt ein Verständnis geschaffen hat. Ab 2013 hat sich nämlich das an der Universität Regensburg, Fakultät für Sprach-, Literatur- und Kulturwissenschaften (SLK), angesiedelte Forschungszentrum ,Deutsch in Mittel-, Ost- und Südosteuropa" (FZDiMOS) unter der Leitung von Prof. Dr. Hermann Scheuringer aktiv und länderübergreifend um die Erforschung der deutschen Sprache im mittleren, östlichen und südöstlichen Europa bemüht. Über gemeinsame Tagungen ${ }^{27}$, Kooperationen und Forschungsprojekte will man zudem auch den menschlichen und fachlichen Austausch unter den Wissenschaftlern in dieser Region fördern. Das FZ DiMOS hat sich nicht nur der Förderung der deutschen Sprache und den deutschen Sprachgemeinschaften in diesem Areal gewidmet, sondern auch ein Forschernetzwerk aufgebaut und die Auslandsgermanistik stark unterstützt.

Erwähnswert sind auch die Beschäftigungen der Hermannstädter mit renommierten siebenbürgisch-rumänischen Publikationen wie Tribuna oder Telegraful român und der Vermittlung des deutschen bzw. rumänischen Kulturguts durch Übersetzungen $^{28}$ deutschsprachiger, auch einheimischer Schriftsteller (z.B.

halb des geschlossenen deutschen Sprachraums. In vielen Mischehen der hier Verbliebenen wird zwar noch Deutsch gesprochen, allerdings oft nur mit einem Elternteil und fast nie Dialekt. Trotz der massiven Auswanderung der deutschsprachigen Minderheit nach dem Ende der Ceauşescu-Diktatur 1989 wird Deutsch in Rumänien und damit auch das Rumäniendeutsche durch Literatur, Schulwesen, Verlagswesen und Presse gefördert. Zu der Gefährdung der Viertelzentren durch sinkende Sprecherzahlen vgl. Ammon, Ulrich: Die Stellung der deutschen Sprache in der Welt. Berlin 2015, S. 341-349.

${ }^{27}$ Vgl. die Programme der bisherigen DiMOS-Tagungen unter http:// www.uni-regensburg.de/forschung/dimos/veranstaltungen/index. html; 04.05.2020.

${ }^{28}$ Vgl. hierzu Sass, Maria: Joachim Wittstock: Forestiera Feltrinelli. Mit şi crâmpeie de realitate. Traducere şi Postfață de Maria Sass. Sibiu 
Joachim Wittstock, Carmen E. Puchianu oder Erika Mitterer) oder das Wirken namhafter Persönlichkeiten zur Rezeption deutscher literarischer Zeugnisse (z.B. George Coşbuc ${ }^{29}$, Ştefan Octavian Iosif oder Lucian Blaga). Die Ergebnisse derartiger und ähnlicher Forschungsvorhaben werden als Monografien, Dissertationen, Beiträge in Sammel- und Tagungsbänden oder Fachzeitschriften veröffentlicht. Unter der Anleitung der im Fachbereich Literatur wirkenden Hochschullehrer schrieben auch 30 Jahre nach der Wende Studierende ihre Abschlussarbeiten zu Themen aus der siebenbürgisch-deutschen Literaturgeschichte (aus interkultureller, übersetzungsrelevanter oder vergleichender Sicht). Und auch nach der Bologna-Reform 2005 blieb die siebenbürgisch-rumäniendeutsche Stofflichkeit in der akademischen Ausbildung erhalten ${ }^{30}$.

Unterstützende Initiativen, vor allem aus Deutschland und Österreich ${ }^{31}$, haben nach dem Umbruch 1990 Forschungsaufent-

2019; Sass, Maria: Joachim Wittstock: Protectoarea dalmată a Păcii. Pagini de proză. Sub redacţia şi cu o postfaţă de prof. univ. dr. Maria Sass. Sibiu 2009; Sass, Maria: Karin Gündisch: Geschichten über Astrid/Povestiri despre Astrid. Ediție bilingvă. București 2017; Sass, Maria: Carmen Elisabeth Puchianu: Confuzie. O poveste pentru copii. In: Cotârlea, Delia (Hg.): Schreiben zwischen Kulturen. Festschrift für Carmen E. Puchianu. Braşov 2017, S. 249-256. Vgl. auch Sass, Maria/ Galter, Sunhild (Hgg.):Schreiben ist zweifellos ein Ziegelrücken. Studien und Aufsätze zu Joachim Wittstocks Werk. Joachim Wittstock: Bibliographie 1999-2009. Germanistische Beiträge 25, Sonderband. Sibiu 2009.

${ }^{29}$ Sass, Maria: Coşbuc şi literatura germană. Sibiu 2000.

${ }^{30}$ An der Hermannstädter Germanistik wurde 2008 das Zentrum für rumäniendeutsche Literatur eingerichtet, dessen Bücher- und Zeitschriftenbestand von den hiesigen Lehrkräften und Nachwuchswissenschaftlern zu Dokumentationszwecken genutzt wird.

${ }^{31}$ Eine vielseitige Unterstützung erfuhrt die Hermannstädter Germanistik durch die Botschaften der Bundesrepublik Deutschland und der Republik Österreich in Bukarest, das Konsulat in Hermannstadt, den Deutschen Wirtschaftsclub, den Stifterverband für die Deutsche Wissenschaft oder den Österreichischen Austauschdienst (OeAD). 
halte $^{32}$ für rumänische Germanisten und Nachwuchswissenschaftler, Gemeinschaftsprojekte, Publikationen (z.B. Tagungsbände) oder sonstige Aktivitäten wie Sommerschulen und Fortbildungsseminare kontinuierlich gesichert. Für den Wissensaustausch, die internationale Zusammenarbeit ${ }^{33}$ und damit auch für die Förderung von Studierenden und Hochschullehrern waren und sind Netzwerke und Partnerschaften zwischen rumänischen und deutschen Universitäten ausschlaggebend ${ }^{34}$. Verschiedenartige Kooperationen in Lehre und Forschung haben der Germanistik in Hermannstadt nach der Wende ihren ,alten Glanz" zurückgegeben und ihr zum Aufschwung verholfen. So hat der ,lebendigsten und erfolgreichsten akademischen Partnerschaft" (1997-2017) der Lucian-Blaga-Universität Hermannstadt mit der Philipps-Universität Marburg ${ }^{35}$ die hiesige

${ }^{32}$ Auf die Erwähnung der Fördermöglichkeiten durch die Vergabe von Stipendien durch diverse akademische Einrichtungen wird hier verzichtet.

${ }^{33}$ Die Lucian-Blaga-Universität in Hermannstadt/Sibiu pflegt Partnerschaften - z.B. Austauschprogramme für Studien- und Forschungsaufenthalte und Projektkooperationen - mit Universitäten aus den USA, Deutschland, Frankreich, Großbritannien, China, Russland, Italien, Griechenland, Polen u.a. Vgl. auch die Homepage: www.ulbsibiu.ro.

${ }^{34}$ Eine Zusammenarbeit mit ausländischen Institutionen gab es bereits vor der Wende, beginnend mit dem von Ulrich Engel initiierten und geleiteten Projekt zur Kontrastiven Grammatik ab Mitte der 1970erJahre. Von 1976 bis 1984 übernahm Prof. Dr. Mihai Isbăşescu die Lehrstuhlleitung der Germanistik in Bukarest. In dieser Zeit wurden Lehrund Forschungsleistungen gefördert, die der langjährigen Zusammenarbeit mit dem Institut für Deutsche Sprache in Mannheim zu verdanken sind.

${ }^{35}$ Vgl. hierzu Konnerth, Gerhard: Die Partnerschaft zwischen der LucianBlaga-Universität Hermannstadt und der Philipps-Universität Marburg. In: Sceculum 37/2012, S. 119-130 und Konnerth, Gerhard: Die Einbindung der Hermannstädter Germanistikin europäische Bezüge. Vortrag bei dem Internationalen Kolloquium: Wirksame Rede im Frühneuhochdeutschen: Syntaktische und textstilistische Aspekte (6. September 2013). In: Germanistische Beiträge 33/2013, S. 11-27. 
Germanistik fachlich und auch menschlich sehr viel zu verdan$\mathrm{ken}^{36}$. Am Lehrstuhl waren zeitweilig zwei Marburger Professoren, Prof. Dr. Monika Rössing-Hager, im Rahmen der Stiftungs-Initiative Johann Gottfried Herder, und Prof. Dr. Heinrich J. Dingeldein als Honorarprofessor, tätig ${ }^{37}$.

Innerhalb dieser Universitätspartnerschaft sind Forschungsprojekte gefördert worden, die - wie bereits oben angedeutet - der regionalen Zuordnung und der Gunst des Standorts Hermannstadt Rechnung trugen. So wurde die Beschäftigung mit älteren Quellentexten ${ }^{38}$ - auch des Zentralarchivs der Evangelischen Kirche A.B. in Rumänien ${ }^{39}$ - von den beiden Marburger Professoren nicht nur angeregt, sondern auch wissen-

${ }^{36}$ Diese Partnerschaft wurde jährlich mit 20.000 Euro vom DAAD gefördert.

${ }^{37}$ Heinrich Dingeldein wurde im Oktober 2007 Doktorandenbetreuer an der Universität Hermannstadt im Fachbereich deutsche Philologie. Über den Stifterverband für die Deutsche Wissenschaft und seine stetige Unterstützung konnten in der damaligen Zeit die Mittel für den Druck der wissenschaftlichen Publikation des Hermannstädter Lehrstuhls, Germanistische Beiträge, zur Verfügung gestellt werden.

${ }^{38}$ Der Dokumentation frühneuhochdeutscher institutioneller Schriftlichkeit im Verwaltungszentrum Hermannstadt aufgrund von Magistratsund Judikatsprotokollen des Verwaltungzentrums Hermannstadt war das vom rumänischen Ministerium für Bildung und Forschung finanzierte Forschungsprojekt Deutschsprachige Kanzleischriftstücke in siebenbürgischen Archiven. Erfassung, Edition, semantische und morphosyntaktische Beschreibung (Projektlaufzeit: 2012-2016) unter der Leitung von Dr. Dana Janetta Dogaru gewidmet.

${ }^{39}$ Das Landeskirchliche Zentralarchiv in Hermannstadt beherbergt Kirchenarchive aus über 280 siebenbürgischen Gemeinden. Infolge der massiven Auswanderung der Deutschstämmigen nach 1990 wurden Gemeindearchive in großer Zahl aufgelöst oder ungeschützt zurückgelassen. Im Archiv werden diese kulturhistorisch bedeutsamen Kulturgüter seitdem gesammelt, erschlossen und für die wissenschaftliche Arbeit zugänglich gemacht. Das Material reicht bis ins Mittelalter zurück und birgt seit der Reformationszeit deutschsprachige Quellen (teils handschriftlich, teils aber auch frühe Drucke). 
schaftlich betreut ${ }^{40}$. Der tatkräftigen finanziellen, wissenschaftlichen und menschlichen Förderung durch diese Partnerschaft ist auch die Entstehung von Dissertationen ${ }^{41} \mathrm{zu}$ verdanken.

Durch die Partnerschaften und die zahlreichen Förderungen in den 1990er-Jahren war einerseits eine inhaltliche Annäherung an die binnendeutsche Germanistik und ihre Aufgaben möglich, andererseits gingen zahlreiche Anstöße und Neuorientierungen von ihr über Kooperationen aus.

Zur Verortung der Hermannstädter Germanistik im Inland hat auch die Fachzeitschrift Germanistische Beiträge (1993 gegründet) ${ }^{42}$ beigetragen. Herausgegeben wurde die Publikation in den ersten Jahren ihres Erscheinens von Gerhard Konnerth, Horst Schuller, Rodica-Ofelia Miclea. Maria Sass hat sich seit 2004 um die Wiederbelebung dieser Fachzeitschrift und um einen Stab an Stammautoren bemüht. Auch damit hat sie sich bleibende Verdienste erworben. Die Germanistischen Beiträge gehören zu den ältesten Publikationen der Fakultät, genauer genommen ist sie die älteste Fachzeitschrift des Instituts, die ab 2005 ohne Unterbrechung erscheint. Bei jeweils zwei Heften

${ }^{40}$ Zur Bedeutung deutscher Sprachinseln für die Sprachgeschichte vgl. Dingeldein, Heinrich J.: Deutsch als Muttersprache in Südosteuropa und ihr Quellenwert für die Sprachgeschichtsschreibung des Deutschen. In: Dósa, Attila/Judit Hell/Gábor Kecskeméti (Hgg.): Beiträge der II. Germanistischen Konferenz: Interdisziplinarität in der Germanistik: AnnäherungeninderLiteratur-, Sprach-undKulturwissenschaft. Universität Miskolc 2009. Miskolc 2010, S. 9-16.

${ }^{41}$ Verwiesen sei hier lediglich auf die erste und auf die letzte Doktorarbeit, die im Kontext dieser langjährigen Kooperation entstanden sind: Dogaru, Dana Janetta: Rezipientenbezug und -wirksamkeit in der Syntax der Predigten des siebenbürgisch-sächsischen Pfarrers Damasus Dürr (ca. 1535-1585). Hildesheim 2006 und Rudolf, Gerhild: Wurzeln und Wege. Soziolinguistische Studie zur kirchlichen Sprachenwahl der Siebenbürger Sachsen. Hermannstadt 2019. Vgl. hierzu auch die Rezension dazu in diesem Heft.

${ }^{42}$ Germanistische Beiträge (ISSN 1454-5144) unter http://uniblaga.eu/ ro/germanistische-beitrage/ einsehbar. 
im Jahr können die Germanistischen Beiträge im Jahr 2020 auf insgesamt 46 Ausgaben stolz sein. Neben Aufsätzen aus dem Fachbereich Germanistik werden in der Fachzeitschrift Rezensionen und nach 2015 zunehmend Tagungsvorträge veröffentlicht.

Die traditionsreiche rumänische Germanistik definiert sich auch durch den besonderen Stellenwert der deutschen Sprache und Kultur hierzulande. Die Germanistischen Beiträge haben in den 25 Jahren ihres Bestehens - in der wissenschaftlichen (fremdsprachlichen) Publikationslandschaft in Rumänien absolut keine Selbstverständlichkeit - Fachartikel unterschiedlicher germanistischer Disziplinen (Sprach-, Literatur-, Kulturwissenschaft und Translatorik sowie Fremdsprachendidaktik) namhafter Vertreter der rumänischen Germanistik veröffentlicht. Ziel dieser Publikation war auch, jungen Nachwuchswissenschaftlern die Möglichkeit zu bieten, ihre Dissertationsvorhaben einem Fachpublikum vorzustellen.

Durch das lange Ausbleiben eines germanistischen Jahrbuchs hierzulande (das unter dem Titel transcarpathica erst 2002 erscheinen konnte) versteht und verstand sich unsere Publikation als Forum hauptsächlich für alle in Rumänien tätigen Germanisten. Darüber hinaus bemüht sich die Publikation um die Förderung der deutschen Kultur und um die Stärkung der Stellung des Deutschen in Rumänien, da in Rumänien Deutsch nicht nur Muttersprache für eine schwindende Bevölkerungsschicht ist, sondern auch Kultursprache, die von Nichtmuttersprachlern gepflegt wird.

Die Germanistischen Beiträgeverdeutlichen Perspektiven, wissenschaftliche Aktivitäten, Forschungstrends und auch Traditionslinien der rumänischen Germanistik, die ihren Nährboden aus der Erforschung der deutschsprachigen regionalen Literatur und Sprache, den Wechselwirkungen mit dem rumänischen Kulturfeld und derEruierung des rumänienspezifischen Umgangs mit Werten, Vorstellungen und Normen der deutschsprachigen 
Kultur speist und damit auch das wichtige kulturelle, sprachliche und geistige Erbe der deutschen Minderheit in Rumänien zu bewahren versucht.

Ab 2019 wird die Publikation über den Verlagsdienstleister $\mathrm{Sciendo}^{43}$, ein Tochterunternehmen von De Gruyter, vertrieben, was die Zitierungsquote und den Einfluss der veröffentlichten Arbeiten erheblich erhöht ${ }^{44}$. De Gruyter ist einer der führenden Verlage für "Open Access" ${ }^{4}$. Sciendo veröffentlicht derzeit rund 600 Zeitschriften. Viele dieser Zeitschriften werden von Clarivate Analytics (ehemals Thomson Reuters), Scopus und PubMed indexiert, wobei einige über hohe Impact Factors verfügen. ${ }^{46}$

Die Internationalisierung, Globalisierung und die durch die Bologna-Reform (1999) ausgelösten Veränderungen haben den Hochschulbetrieb insgesamt und damit auch die inhaltliche und organisatorische Ausrichtung der Studiengänge im Fachbereich Germanistik beeinflusst und dies nicht nur an rumänischen Universitäten. Fachvertreter unterschiedlicher Länder verweisen auf curriculare Weiterentwicklungen und damit auf die verstärkte Ausrichtung der Germanistik auf Interdisziplinarität und Arbeitsmarktorientierung, auf die notwendige Kooperation mit der Wirtschaft oder auf künftige Ausbildungsinhalte

${ }^{43}$ Neuer Verlagsdienstleister für die Wissenschaft. Beitrag vom 14.05.2018; unter https://www.boersenblatt.net/2018-05-14-artikelde_gruyter_startet_sciendo_-neuer_verlagsdienstleister_fuer_die wissenschaft. $1467987 . \mathrm{html} ; \overline{4} .5 .202 \overline{0}$.

${ }^{44}$ Näheres auf der Homepage von Sciendo unter https://www.degruyter. com/page/open-access; 04.05.2020.

${ }^{45}$ Der Verlag, der über 270 Jahre verlegerische Erfahrung hat, veröffentlicht jährlich über 1300 neue Buchtitel und mehr als 900 Zeitschriften in den MINT-Fachgebieten (Mathematik, Informatik, Naturwissenschaften, Technik) sowie in den Geistes- und Sozialwissenschaften (GSW) im Open-Access-Format. Vgl. die Homepage unter https:// www.degruyter.com/?language $=$ de; 4.5.2020.

${ }^{46} \mathrm{Vgl}$. https://content.sciendo.com/view/journals/gb/gb-overview.xml; 4.5.2020. 
und -ziele im Lehramt Deutsch. ${ }^{47}$ Landesweit wurden nach 2005 Anstrengungen unternommen, die Lehrpläne den BolognaBeschlüssen anzupassen $^{48}$. Darüber hinaus haben bildungspolitische Entscheidungen, darunter die Minderung der Stundenanzahl für den Fremdsprachenunterricht an rumänischen Schulen, den Status des Deutschen geschwächt und die Fremdsprachenwahl an Schulen und Hochschulen gesteuert, damit die Dominanz von Englisch begünstigt.

Kurz nach der Jahrtausendwende begann sich ein Wandel in der traditionellen Germanistik abzuzeichnen, der sich auch im Aufkommen arbeitsmarktorientierter und praxisnaher Studienangebote äußerte. Bildungspolitische Reformen sind gefordert und durchgesetzt worden, wobei die Vermittlung berufsorientierter Qualifikationen und eine gesteigerte Arbeitsanschlussfähigkeit bei der Konzipierung des Studienangebotes vordergründig waren. Folglich musste auch die philologische Ausbildung verstärkt auf Interdisziplinarität, Berufsbezug und den Erwerb von Deutsch als Fach- und Berufssprache ausgerichtet sein. Dieser Wandel war auch eine Folge erweiterter Berufsaussichten und veränderter Ansprüche der Studierenden, die arbeitsmarktrelevantes Wissen und eine breitere Kompetenzvermittlung in der universitären Germanistik-Ausbildung

${ }^{47}$ Vgl. hierzu auch den Sammelband von Middeke, Annegret/Sava, Doris/ Tichy, Ellen (Hgg.): Germanistische Diskurs- und Praxisfelder in Mittelosteuropa. Berlin 2019. Der Sammelband bringt eine Bestandsaufnahme der Entwicklungen des Faches Germanistik aus der Sicht ihrer interdisziplinären Ausrichtung und Orientierung auf neue Berufsbilder in der Region Mittelost- und Südosteuropas. Fokussiert wird auf bildungspolitische und curriculare Wandelprozesse sowie auf die Herausforderungen der internationalen Germanistik nach den ersten zwei Dekaden des neuen Jahrtausends. Aus unterschiedlichen Perspektiven wird somit die konkrete Situation der Germanistik und der Fremdsprache Deutsch an (Hoch-)Schulen und Universitäten in Rumänien, Serbien, Ungarn und Polen erfasst, um den Wandel des Faches und den veränderten Status von Deutsch zu dokumentieren.

${ }^{48}$ In Rumänien wurde die Bologna-Reform 2005 umgesetzt. 
erwarteten ${ }^{49}$. Neben den klassischen Germanistikstudiengängen wurden interdisziplinäre Studiengänge angeboten, die am Arbeitsmarkt orientiert sind, und die in Konkurrenz zu den herkömmlichen, traditionellen Studienangeboten ${ }^{50}$ stehen.

Das traditionelle Rahmencurriculum im Fachbereich Germanistik umfasst Lehrveranstaltungen zur Linguistik, Literaturwissenschaft, Landeskunde und Didaktik sowie Wahlfächer und Seminare zur Verbesserung der Sprachkompetenz (rum. curs practic oder practica limbii). Das philologische Studium bildet vornehmlich für sprach- und kulturbezogene Berufe in Schulen, Bildungseinrichtungen, im Verlags- und Bibliothekswesen, im Bereich der Medien, des Kultur- und Wissenschaftsmanagements, in der interkulturellen Kommunikation usw. aus. Abgänger diverser Hochschulstudiengänge bevorzugen jedoch, falls sie sich für den Verbleib in Rumänien und nicht für die Jobsuche im EU-Raum entscheiden, was nach der Wende, besonders verstärkt jedoch nach dem EU-Beitritt Rumäniens 2007, der Fall ist, weniger eine Stelle im Lehramt oder in der Wissenschaft, sondern einen Arbeitsplatz in einem deutschsprachigen

${ }^{49}$ Gegenwärtig wird Germanistik als Studienfach an zehn staatlichen Universitäten und an drei privaten Universitäten angeboten.

${ }^{50} \mathrm{Zu}$ den gegenwärtigen deutschsprachigen Studienangeboten an rumänischen Universitäten vgl. auch Cosma, Ruxandra/Koch, Marianne: Bericht zur Lage der Germanistik in Rumänien. In: Brdar-Szabó, Rita/Elisabeth Knipf-Komlósi/Roberta V. Rada (Hgg.): Zur Rolle und Positionierung des Deutschen in Mittelosteuropa. Sprachpolitische Überlegungen. Konferenzband zur Tagung Deutsch 3.0 in Budapest am 15-16. Mai 2014. Budapest 2014, S. 130-145; Galter, Sunhild: Studiengänge der Germanistik. Entwicklungen und Tendenzen. In: Tichy, Ellen/Viktoria Ilse (Hgg.): Deutsch in Mittelosteuropa nach 1989. 25 Jahre Germanistikstudiengänge, Deutschlehrerausbildung, DaF-Lehrwerke und DaF-Unterricht. Frankfurt/Main 2014, S. 129138 und Galter, Sunhild: Die Germanistikabteilungen an rumänischen Universitäten im Wandel der Zeit. In: Middeke, Annegret/Doris Sava/ Ellen Tichy (Hgg.): Germanistische Diskurs- und Praxisfelder in Mittelosteuropa. Berlin 2019, S. 15-39. 
Unternehmen ${ }^{51}$. Dabei spielen gute Sprachkenntnisse eine Rolle, sodass sich Studierende vornehmlich für den Sprachunterricht und weniger für die Vermittlung deutschsprachiger Literatur interessieren.

Bei der Umsetzung der Bologna-Reformen am hiesigen Lehrstuhl für Germanistik blieb die Lehrerausbildung weiterhin vordergründig, allerdings wurden die Curricula umgestaltet, sodass den Studierenden Schlüsselqualifikationen und berufliche Kompetenzen vermittelt wurden, die einen (sofortigen) Einstieg in die Berufstätigkeit ermöglichen sollen. ${ }^{52}$ Die stark verminderte Nachfrage nach einem traditionellen Germanistikstudium bei Studienbewerbern mit guten Sprachkenntnissen führte einerseits zur Einrichtung berufsorientierter Studiengänge, die parallel zu den klassischen Germanistikstudiengängen angeboten wurden. ${ }^{53}$ Andererseits haben die meisten Lehrstühle für Germanistik hierzulande die Aufnahmeprüfung nach und nach abgeschafft, um die Anzahl der Studienbewerber auch für das Germanistik-Studium zu erhöhen. Folglich wurden auch

${ }^{51}$ In Rumänien sind ca. 8.000 Unternehmen mit deutscher Beteiligung aktiv. Bewerbende mit guten Deutschkenntnissen sind daher sehr gefragt. Vgl. http://rumaenien.ahk.de/fileadmin/ahk_rumaenien/Publica tii/Presse/2017/Germania.Informatii_generale.pdf; 11.09 .2019 . Zu der Zusatzqualifikation „Deutschkenntnisse“ und den beruflichen Perspektiven der Germanistik-Abgänger allgemein vgl. ebenfalls den Sammelband von Middeke/Sava/Tichy (2019).

${ }^{52}$ Die Hermannstädter Germanistik deckt alle Ausbildungsstufen ab: zwei "Bachelor"-Studiengänge (Germanistik/Philologie und Angewandte Fremdsprachen), zwei Masterstudiengänge (Deutsche Sprache und Literatur, Interkulturelle WirtschaftskommunikationDeutsch) und Doktorat im Fachbereich Philologie (Literaturwissenschaft ab 2009 und Sprachwissenschaft ab 2015).

${ }^{53}$ So wurde z.B. in Bukarest, Klausenburg und Hermannstadt der Studiengang „Angewandte Fremdsprachen“ (rum. Limbi moderne aplicate) gegründet, der auf die Vermittlung von Deutsch als Berufs- und Fachsprache in den Bereichen Wirtschaft, Jura oder Verwaltung bzw. auf das Trainieren des Dolmetschens und des Übersetzens ausgerichtet ist. 
Studienanwärter mit A1- oder Null-Sprachkenntnissen für das Studium zugelassen, da die Finanzierung vorwiegend nach der Studierendenanzahl (Pro-Kopf-Finanzierung) erfolgt ${ }^{54}$. Dass viele Studierende den sprachlichen Anforderungen nicht gewachsen waren, muss hier nicht betont werden. Um einen Studienabbruch zu verhindern, waren die Lehrkräfte genötigt, Rumänisch als Unterrichtssprache einzubeziehen und intensiven, kostenlosen Deutschunterricht sowie zusätzliche sprachpraktische Lehrveranstaltungen anzubieten. In den Lehrveranstaltungen wurde zudem dem Niveau der Studierenden (Fortgeschrittene und Anfänger) entsprechend gesondert unterrichtet. Die Heterogenität der Gruppenzusammensetzung verhinderte demnach einen sprachlich anspruchsvolleren Unterricht und eine eingehendere wissenschaftliche Ausbildung. Zudem ist das Interesse der Germanistik-Abgänger an der universitären Lehre und Forschung stark gesunken und auch die Promotionsplätze wurden aufgrund finanzieller Engpässe eingeschränkt. ${ }^{55}$

Fasst man die dritte Mission der Universität ins Auge als Verpflichtung, sich für das wirtschaftliche und gesellschaftliche Umfeld zu öffnen, so nehmen in der Hochschulpolitik Kooperationen mit anderen Einrichtungen und der Wirtschaftswelt eine wichtige Stellung ein. Das Postulat einer weitgehenden Kompetenzorientierung in der akademischen Lehre durch die Vermittlung von Schlüsselkompetenzen für den Berufsalltag und die angestrebte Arbeitsmarktorientierung wurden als Entwicklungen gewertet, die zur Ent-Philologisierung des Faches geführt haben ${ }^{56}$.

${ }^{54}$ Aufgrund der sinkenden Nachfrage werden philologische Studienbereiche vom Staat unterfinanziert. Auch können Universitäten aus finanziellen Gründen qualifiziertes Personal für die akademische Lehre und Forschung kaum anwerben oder fördern.

${ }^{55}$ Ein Promotionsabschluss im Fachbereich Germanistik ist an den Universitäten in Bukarest, Iasi, Cluj, Sibiu, Braşov und Timișoara möglich.

${ }^{56}$ Zum Wandel in der rumäniendeutschen Germanistik vgl. auch Konnerth (2013: 19): „Die rumänische Hochschulgermanistik versteht sich heute 
Das neue Unterrichtsgesetz 2010 brachte die Pro-KopfFinanzierung und die Einrichtungen von Departements. Aufgrund bildungspolitischer Entscheidungen wurden 2011 die Germanistik und Anglistik als eigenständige Lehrstühle administrativ aufgelöst und in ein Departement umgewandelt. Dieser Meilenstein in der jüngsten Geschichte der Germanistik hattenegative Folgen fürdieGermanistik. DerZusammenschluss zu einem "Mammut"-Departement mit fast 40 Mitgliedern ließ nur zögerlich eine wirksame menschliche und wissenschaftliche Zusammenarbeit zustandekommen. Nicht nur, dass sich die Mitarbeiter der ehemaligen Lehrstühle voneinander isoliert hatten und die Germanisten mit den Anglisten auch auf menschlicher Ebene kaum auskamen, auch die Ressourcen des Departements insgesamt wurden knapper. Absurde drastische Sparmaßnahmen, rhetorische Effekthascherei und falsches Konkurrenzdenken erschwerten den Austausch. Die Germanistik stand - zugespitzt formuliert - vor der Herausforderung, ihre Stellung zu stärken, um die traditionellen Schwerpunkte ihres Wirkens zu wahren. Diese Kopplung brachte für die sowieso vom Staat stiefmütterlich behandelte Germanistik vielfältige Schwierigkeiten und auch ein gewisser Verlust an Qualität.

Es bedarf keines Blicks in die Zukunft, um Gewissheit zu haben: Vieles hat sich verändert. Wohin steuert also unser Fach? ${ }^{57}$

als „eine Auslandsgermanistik, die ihre Schwerpunkte neu definiert“. Vgl. hierzu auch Sass, Maria: Die Hermannstädter Germanistik: Rückschau und Perspektiven. In: Tichy, Ellen et al. (Hgg.): Germanistik und Wirtschaft in der Diskussion. Tagungsband: Kulturelle Zentren der deutschen Minderheiten und berufliche Perspektiven in deutschsprachigen Wirtschaftsunternehmen. Berlin 2018, S. 49-58.

${ }^{57}$ Reflexionen zur Entwicklung des traditionellen Faches Germanistik sind auf der Jubiläumstagung Germanistik im Spiegel: Wege und Umwege einer Wissenschaft (Klausenburg, 13.-14. Juni 2019) anlässlich des 100-jährigen Bestehens des Klausenburger Lehrstuhls für Germanistik ausgetragen worden. Weitaus früher veranstaltete z.B. die kleine Germanistikabteilung des damaligen Lehrstuhls für Fremdsprachen und -literaturen an der Transilvania-Universität in Kronstadt 
Mit der wettbewerblichen Ausrichtung der Universitäten, die auf dem globalen Bildungsmarkt um Studierende, Geldmittel, wissenschaftliche Sichtbarkeit und eine bessere Position in internationalen Rankings miteinander konkurrieren, gehen auch „moderne“ Instrumente der Qualitätssicherung und -überprüfung einher ${ }^{58}$.

Trotz Kursänderung infolge weltweiter Wandlungen des Selbstverständnisses und Neuorientierungen - externe Evaluierungen infolge der Reform der Studiengangstruktur und der Einführung gestufter Studiengänge, Senkung der Zugangsvoraussetzungen, niedrigere Anforderungen an Studierende, mangelnde Attraktivität des Lehrerberufs, rückläufige Einschreibungen, steigendeArbeitsmarktorientierungundKompetenzorientierung, Öffnung zur außeruniversitären Berufswelt in den Curricula germanistischer Studiengänge bzw. Schwinden und Verlust des herkömmlichen philologischen Anteils in den Curricula - werden gut ausgebildete Germanisten in mehreren Tätigkeitsfeldern exzellente Perspektiven haben: durch gute Sprachkenntnisse und im Studium erworbene Fertigkeiten für die Praxis.

im Zeitraum 21.-24. März 2002 ihre IV. Jahrestagung zum Thema: Germanistik im dritten Millenium - ungeahnte Möglichkeiten oder Sackgasse?

${ }^{58}$ Seit Mitte der 1990er-Jahre lässt sich eine verstärkte Internationalisierung von Hochschulen beobachten, auch in Rumänien. Hier wurden auslandsorientierte Studiengänge eingerichtet, in denen vorwiegend in einer Fremdsprache unterrichtet wird oder es wurden gemeinsame Studiengänge mit ausländischen Partneruniversitäten konzipiert, die häufig mit einem Doppeldiplom abgeschlossen werden. Zur Internationalisierung gehören auch die Modularisierung der Studieninhalte, die Vergabe von auch in anderen Ländern anerkannten Kreditpunkten sowie das sogenannte „Diploma Supplement“. Auch ist die internationale Hochschulkooperation und Mobilität von Studierenden und Lehrenden durch die Einrichtung des ERASMUS-Programms stark gefördert worden. An der Universität Hermannstadt bestehen Kooperationen mit Einrichtungen in Deutschland, Österreich, Ungarn, Slowenien oder Polen. 
Infolge der veränderten Publikationspraxis, die sich in der vermehrten Einreichung von englischsprachigen Aufsätzen für Zeitschriften wichtiger Datenbanken äußert, kann sich der lokale oder nationale wissenschaftliche Publikationsmarkt schwer erhalten. Selbst von den einheimischen Universitäten werden Veröffentlichungen in möglichst hochgerankten anglo-amerikanischen Zeitschriften eingefordert, deren Publikationssprache fastausschließlich Englisch ist. Fachpublikation derGermanistik in Rumänien gibt es an allen traditionsreichen germanistischen Standorten (Timișoara, Oradea, Cluj-Napoca, Sibiu, Brașov, Iași und Bukarest). Durch die Förderung eigener Lehr- und Forschungstraditionen und spezifischer Themenbereiche - Geschichte und Kulturtradition der deutschsprachigen Bevölkerung in Siebenbürgen, im Banat, in der Bukowina, in der Dobrudscha sowie in Bukarest, Kulturaustausch in multiethnischen Gebieten, identitätsstiftende Traditionen - haben diese Publikationen, deren Publikationssprache Deutsch ist, den Ruf der rumänischen (Auslands)Germanistik gestärkt. Ein wichtiger und intensiv gepflegter Forschungsbereich der Hermannstädter Germanistik stellt die rumäniendeutsche Literatur dar. Die Lehrstuhlpublikation Germanistische Beiträge widmet sich daher vornehmlich den Entwicklungssträngen und charakteristischen Merkmalen dieser Literatur, wobei die Ergebnisse der interdisziplinär und interkulturell ausgerichteten Forschungstätigkeit rumänischer und deutscher Germanisten ebenfalls berücksichtigt werden. Die Tatsache, dass die Forschungsergebnisse rumänischer Germanisten und Forscher aufDeutsch und auch in den Hermannstädter Germanistischen Beiträgen veröffentlicht werden, ist ein wichtiger Beitrag zur Aufrechterhaltung und Förderung des Deutschen als Wissenschaftssprache in diesem Teil Europas.

In dieser feierlichen Stunde gebührt Dank allen Mitarbeitern von nah und fern, treue Weggefährten und Freunde, frühere und gegenwärtige Fachkollegen, die unseren Lehrstuhl tatkräftig auch finanziell - unterstützt und dessen Publikation durch Jahre 
hindurch mit Materialien (Aufsätze, Rezensionen, Berichte) versorgt haben. Von den ausländischen Förderern und Mitarbeitern seien Stefan Sienerth ${ }^{59}$, Heinrich Dingeldein und Eugen Christ ${ }^{60}$ erwähnt, von den hiesigen Sigrid Haldenwang ${ }^{61}$, Gerhild

${ }^{59}$ Der namhafte Germanist, Literaturgeschichtler und -wissenschaftler Prof. Dr. Dr. h.c. Stefan Sienerth, exzellenter Kenner und Förderer deutscher Regionalliteraturen Südosteuropas, der regionalen Literaturgeschichtsschreibung, dersiebenbürgisch-deutschenLiteraturgeschichte und des deutschsiebenbürgischen Schrifttums wie auch der siebenbürgisch-sächsischen Mundartlexikografie kam 1975 an den hiesigen Lehrstuhl. 1979 promovierte Stefan Sienerth an der Universität Bukarest. Als der Germanistiklehrstuhl aufgelöst wurde, kann Stefan Sienerth als Mundartsprecher am Siebenbürgisch-Sächsischen Wörterbuch (SSWB) beim damaligen Forschungsinstitut für Sozial- und Geisteswissenschaften in Hermannstadt (1986-1990) als wissenschaftlicher Mitarbeiter mitwirken. An der Hermannstädter Forschungseinrichtung war er zudem Redaktionssekretär und Mitglied im Redaktionskollegium der Zeitschrift Forschungen zur Volks- und Landeskunde. Stefan Sienerth hat auch nach seiner Ausreise 1990 die Vermittlung der siebenbürgisch-deutschen und rumäniendeutschen Literatur bis in die Gegenwart engagiert weitergeführt und sich für deren Wahrnehmung und Förderung resolut eingesetzt. Auch gegenwärtig ist Stefan Sienerth, dessen Sachkompetenz mehreren Bänden des SSWB zugute kam, diesem Standardwerk der siebenbürgisch-sächsischen Mundartforschung treu geblieben.

${ }^{60}$ Dr. Eugen Christ ist Geschäftsführer der Donauschwäbischen Kulturstiftung des Landes Baden-Württtemberg (DSKS). Zu den Aktivitäten vgl. auch die Homepage unter http://www.dsksbw.de/; 04.05.2020.

${ }^{61}$ Dr. Sigrid Haldenwang ist als wissenschaftliche Mitarbeiterin ersten akademischen Grades an der Forschungsstelle zum SiebenbürgischSächsischen Wörterbuch (SSWB), Standardwerk und Sprachdenkmal zugleich, am Forschungsinstitut für Geisteswissenschaften Hermannstadt der Rumänischen Akademie tätig. Von den hundert Jahren Wörterbuchgeschichte ist Sigrid Haldenwang seit fünf Jahrzehnten dabei. Unter ihrer Mitwirkung entstanden am Institut die Lieferungen von 6 Bänden des SSWB [Bd. 5 (K) 1975; Bd. 6 (L) 1993; Bd. 7 (M) 1998; Bd. 8 (N-P) 2002; Bd. 9 (Q-R) 2006; Bd. 10 (S-Sche) 2014]. Alle ehemaligen Bearbeiter der Wörterbuchstelle sind ausgereist, Sigrid 
Rudolf $^{62}$ und Joachim Wittstock. Ihr freundliches Entgegenkommen, ihre Austauschbereitschaft und Freundschaft haben uns auch menschlich bereichert. Unser Dank geht auch an alle Mitwirkenden an diesem Sonderband, der die Vorträge zur 50. Jubiläumstagung vereint. Es finden sich darunter langjährige Mitarbeiter der Germanistichen Beiträge und alljährliche Tagungsgäste, deren wohlwollende Unterstützung und Verbundenheit wir ebenfalls zu schätzen wissen: Ioana Crăciun und Markus Fischer (Universität Bukarest), Carmen E. Puchianu ${ }^{63}$ und Delia

Haldenwang blieb jedoch, ihrem“Wörterbuch treu. Den Sprachsschatz dieser deutschen Sprachinsel lexikografisch zu erfassen und der Nachwelt zu erhalten, ist ihre Lebensaufgabe. Dr. Sigrid Haldenwang ist eine langjährige Mitarbeiterin der Germanistischen Beiträge, in der sie ab 1995 kontinuierlich Aufsätze veröffentlicht hat.

${ }^{62}$ Dr. Gerhild Rudolf ist seit 2012 Leiterin des Begegnungs- und Kulturzentrums „Friedrich Teutsch“ der Evangelischen Kirche A. B. in Rumänien (EKR).

${ }^{63}$ Seit 2018 Geschäftsführende Vorsitzende der Gesellschaft der Germanisten Rumäniens (GGR). Die Gründung der Gesellschaft der Germanisten Rumäniens (GGR) ermöglichte eine landesweite Kooperation der einzelnen Germanistiklehrstühle, die sich auch in der Neubelebung der germanistischen Forschung äußerte. Prof. Dr. George Guțu, Bukarester Hochschullehrer und Leiter des Germanistiklehrstuhls (1998-2011), hat die germanistische Tradition Rumäniens aus der Zwischenkriegszeit fortgeführt und im Jahre 1990 die GGR gegründet. Ihm ist auch die Wiederaufnahme einer weiteren Tradition - nach 62 Jahren! - zu verdanken: die Gründung der Zeitschrift der Germanisten Rumäniens (1992) und die seit 1994 regelmäßig alle drei Jahre stattfindenden internationalen Kongresse der Germanisten Rumäniens. Für die Anerkennung und Schätzung seines langjährigen Beitrags zur Förderung der deutschen Kultur und Literatur im Bildungs- und im Forschungsbereich, der Kulturkontakte zwischen Rumänien und Deutschland bzw. Österreich wurde 2011 George Guţu, Gründer, Präsident (1990-2017) und gegenwärtig Ehrenpräsident der GGR, mit dem Orden für Kulturelle Verdienste im Rang eines Ritters und mit dem Österreichischen Ehrenkreuz für Wissenschaft und Kunst ausgezeichnet. 
Cotârlea (Transilvania-Universität Brasov), Roxana Nubert ${ }^{64}$ und Grazziella Predoiu (West-Universität Timisoara) und Adina-Lucia Nistor (Alexandru-Ioan-Cuza-Universität Iasi).

Allen widrigen Umständen - verhärtete Einstellungs- und Beförderungsbedingungen und strengere Voraussetzungen für die Zuweisung staatlicher Finanzmittel, Bologna-Belastungen infolge curricularer Umstrukturierungen und der Forderung nach verstärkter Berufsbezogenheit, die Ökonomisierung und chronische Unterfinanzierung des Bildungswesens oder sinkende Sprachkenntnisse der Studierenden - zum Trotz ist es dem individuellen Engagement der Hochschullehrkräfte auch aus Hermannstadt zu verdanken, dass die traditionsreiche rumänische Germanistik Beachtliches - die Erkundung eigener, spezifischer Forschungsgebiete und die Bewahrung literarischer Zeugnisse der Rumäniendeutschen - geleistet hat und durch die Veröffentlichung zahlreicher Studien, Übersetzungen oder Wörterbücher die Grundlage für das Studium der Germanistik in Rumänien geschaffen haben.

\section{$* * *$}

Dem festlichen Anlass passend schließe ich diesen Beitrag mit einem abgewandelten irischen Segenswunsch - Möge die hiesige Germanistik stets einen Freund an ihrer Seite haben, der ihr Vertrauen gibt, wenn es ihr an Licht und Kraft gebricht und zuversichtlichen Ausblick: Es gibt sie noch an vereinzelten germanistischen Standorten, die Idealisten, die mit Hingabe die Germanistik betreuen. Und es ist zu hoffen, dass sie auch weiterhin die Energie aufbringen können, um sich den neuen Konstellationen und Belastungen durch Initiativen und organisatorisches Können zu stellen und sich weiterhin für die Belange der Germanistik einzubringen. Denkt man an die

${ }^{64}$ Seit 2018 Präsidentin der Gesellschaft der Germanisten Rumäniens (GGR). 
unausweichlichen Schwierigkeiten des Neuanfangs nach 1990 und des späteren Werdegangs sind Gemeinschaftsempfinden, Traditionspflege, Pflichtgefühl und Handlungsbereitschaft es weiterhin wert, getragen und eingesetzt zu werden.

Die hier vorgeführten Bestrebungen lassen den Schluss zu, dass die Pflege des deutschen Kulturguts auch in Hermannstadt einer Tradition verpflichtet ist, die dem hiesigen Lehrstuhl Anerkennung eingebracht hat. Dieser Erfolg, mit Enttäuschungen und mit Freude verbunden, ist größtenteils dem persönlichen Einsatz, Fleiß und Durchsetzungswillen der jeweiligen Lehrstuhlinhaber und einiger unermüdlichen Idealisten zu verdanken. Aus Anlass des doppelten Geburtstages hat daher mein Dank allen zu gelten, die sich um diesen Lehrstuhl verdient gemacht haben. Mit Blick auf das Jubiläum und auf die im Titel eingebrachte „Wetterkarte“ ließe sich der obige Segenswunsch ein weiteres Mal abwandeln: Mögen alle Verantwortlichen, die die Geschicke der Hermanistik künftig leiten werden, ihr freundlich gewogen sein.

\section{Literatur}

Ammon, Ulrich/Bickel, Hans/Lenz Alexandra N. (Hgg.): Variantenwörterbuch des Deutschen. Die Standardsprache in Österreich, der Schweiz, Deutschland, Liechtenstein, Luxemburg, Ostbelgien und Südtirol sowie Rumänien, Namibia und Mennonitensiedlungen. Berlin 2016.

Ammon, Ulrich: Die Stellung der deutschen Sprache in der Welt. Berlin 2015, S. 341-349.

Baghiu, Ștefan/Pojoga, Vlad/Sass, Maria (Hgg.): Ruralism and Literature in Romania. Berlin 2019.

Bottesch, Johanna: Rumänien. In: Eichinger, Ludwig M./ Albrecht Plewnia/Claudia Maria Riehl (Hgg.): Handbuch derdeutschen Sprachminderheiten in Mittel-und Osteuropa. Tübingen 2008, S. 329-392. 
Cosma, Ruxandra/Koch, Marianne: Bericht zur Lage der Germanistik in Rumänien. In: Brdar-Szabó, Rita/Elisabeth Knipf-Komlósi/Roberta V. Rada (Hgg.): Zur Rolle und Positionierung des Deutschen in Mittelosteuropa. Sprachpolitische Überlegungen. Konferenzband zur Tagung Deutsch 3.0 in Budapest am 15-16. Mai 2014. Budapest 2014, S. 130-145.

Dingeldein, Heinrich J.: Deutsch als Muttersprache in Südosteuropa und ihr Quellenwert für die Sprachgeschichtsschreibung des Deutschen. In: Dósa, Attila/Judit Hell/ Gábor Kecskeméti(Hgg.): Beiträge der II. Germanistischen Konferenz: Interdisziplinarität in der Germanistik: Annäherungen inder Literatur-, Sprach-und Kulturwissenschaft. Universität Miskolc 2009. Miskolc 2010, S. 9-16.

Dogaru, Dana Janetta: Rezipientenbezug und-wirksamkeit in der Syntax der Predigten des siebenbürgisch-sächsischen Pfarrers Damasus Dürr (ca. 1535-1585). Hildesheim 2006.

Galter, Sunhild: Die Germanistikabteilungen an rumänischen Universitäten im Wandel der Zeit. In: Middeke, Annegret/ Doris Sava/Ellen Tichy (Hgg.): Germanistische Diskursund Praxisfelder in Mittelosteuropa. Berlin 2019, S. 15-39.

Galter, Sunhild: Studiengänge der Germanistik. Entwicklungen und Tendenzen. In: Tichy, Ellen/Viktoria Ilse (Hgg.): Deutsch in Mittelosteuropa nach 1989. 25 Jahre Germanistikstudiengänge, Deutschlehrerausbildung, DaF-Lehrwerke und DaF-Unterricht. Frankfurt/Main 2014, S. 129-138.

Galter, Sunhild/Sass, Maria/Tichy, Ellen (Hgg.): Wechselwirkungen im deutsch-rumänischen Kulturfeld. Beiträge zu Sprach- und Literaturkontakten aus interkultureller Perspektive. Frankfurt/Main 2015.

Konnerth, Gerhard: Die Einbindung der Hermannstädter Germanistikin europäische Bezüge. Vortrag bei dem Internationalen Kolloquium: Wirksame Rede im Frühneuhochdeutschen: Syntaktische und textstilistische Aspekte (6. September 2013). In: Germanistische Beiträge 33/2013, S. 11-27. 
Konnerth, Gerhard: Die Partnerschaft zwischen der LucianBlaga-Universität Hermannstadt und der Philipps-Universität Marburg. In: Sceculum 37/2012, S. 119-130.

Konnerth, Gerhard: Der Hermannstädter Lehrstuhl für Germanistik. Ausblick und Rückschau. In: Germanistische Beiträge 13/14, 2000, S. 18-31.

Lăzărescu, Ioan: Rumäniendeutsch - eine eigenständige, jedoch besondere Varietät der deutschen Sprache. In: SchneiderWiejowski, Karina/Birte Kellermeier-Rehbein/Jakob Haselhuber (Hgg.): Vielfalt, Variation und Stellung der deutschen Sprache. Berlin 2013, S. 369-389.

Middeke, Annegret/Sava, Doris/Tichy, Ellen(Hgg.): Germanistische Diskurs- und Praxisfelder in Mittelosteuropa. Berlin 2019.

Miladinović Zalaznik, Mira/Sass, Maria/Sienerth, Stefan (Hgg.): Literarische Zentrenbildung in Ostmittel- und Südosteuropa: Hermannstadt/Sibiu, Laibach/Ljubljana und weitere Fallbeispiele. München 2010.

Rudolf, Gerhild: Wurzeln und Wege. Soziolinguistische Studie zur kirchlichen Sprachenwahl der Siebenbürger Sachsen. Hermannstadt 2019.

Sass, Maria: Joachim Wittstock: Forestiera Feltrinelli. Mit şi crâmpeie de realitate. Traducere şi Postfață de Maria Sass. Sibiu 2019.

Sass,Maria/Baghiu, Ștefan/Pojoga, Vlad (Hgg.): The Culture of Translation in Romania/Übersetzungskultur und Literaturübersetzen in Rumänien. Berlin, 2018.

Sass, Maria: Karin Gündisch: Geschichten über Astrid/Povestiri despre Astrid. Ediție bilingvă. București 2017.

Sass, Maria/Sava, Doris/Sienerth, Stefan (Hgg.): Schriftsteller versus Übersetzer. Begegnungen im deutsch-rumänischen Kulturfeld. Frankfurt/Main 2013.

Sass, Maria: Germanistische Beiträge Monographien: Beiträge germanistischer Nachwuchskräfte, Bd. I-IV. Sibiu 20112014. 
Sass, Maria: Joachim Wittstock: Protectoarea dalmată a Păcii. Pagini de proză. Sub redacţia şi cu o postfaţă de prof. univ. dr. Maria Sass. Sibiu 2009.

Sass, Maria/Galter, Sunhild (Hgg.): Schreiben ist zweifellos ein Ziegelrücken. Studien und Aufsätze zu Joachim Wittstocks Werk. Joachim Wittstock: Bibliographie 1999-2009. Germanistische Beiträge 25, Sonderband. Sibiu 2009.

Sass, Maria: Coşbuc şi literatura germană. Sibiu 2000.

Sass, Maria: Die Hermannstädter Germanistik: Rückschau und Perspektiven. In: Tichy, Ellen et al. (Hgg.): Germanistik und Wirtschaft in der Diskussion. Tagungsband: Kulturelle Zentren der deutschen Minderheiten und berufliche Perspektiven in deutschsprachigen Wirtschaftsunternehmen. Berlin 2018, S. 49-58.

Sass, Maria: Carmen Elisabeth Puchianu: Confuzie. O poveste pentru copii. In: Cotârlea, Delia (Hg.): Schreiben zwischen Kulturen. Festschrift für Carmen E. Puchianu. Braşov 2017, S. 249-256.

Sass, Maria: Tradition heißt nicht, die Asche aufheben, sondern die Flamme weiterreichen: Die Sommerakademie „Siebenbürgen“ und ihr Beitrag zur Förderung der deutschen Sprache, Kultur und des Austausches in Südosteuropa. In: Germanistische Beiträge 33/2013, S. 225-244.

Sass, Maria: Deutsche Sprache, Literatur und Kultur in Rumänien - Bilanz und Perspektiven. In: Christ, Eugen/ Peter Dines/Stefan Jeuk (Hgg.): 25 Jahre Donauschwäbische Kulturstiftung des Landes Baden-Württenberg (19882013). Bruchsal 2013, S. 43-52, 2013.

Wittstock, Joachim: Aus dem Bereich von „Litteris et Artis“. Literarhistorische Erörterungen im Rahmen der Hermannstädter Hochschulgermanistik. In: Germanistische Beiträge 26/2010, S. 15-30. 


\section{Internetquellen}

Fischer, Markus: 50 Jahre Germanistik in Hermannstadt. Internationale Jubiläumstagung an der Lucian-Blaga-Universität. In: Allgemeine Deutsche Zeitung für Rumänien, 4.11.2019; unterhttps://adz.ro/artikel/artikel/50-jahre-germanistik-inhermannstadt; 11.12.2019.

Neuer Verlagsdienstleister für die Wissenschaft. Beitrag vom 14.5.2018; unter https://www.boersenblatt.net/2018-0514-artikel-de_gruyter_startet_sciendo_-neuer_verlagsdienstleister_fuer_die_wissenschaft.1467987.html; 4.5.2020.

Ungar, Beatrice: „Eine zuversichtliche Gemeinschaft“: 50.

Gründungsjubiläum der Germanistik in Hermannstadt. In: Hermannstädter Zeitung, 25.10.2019; unter https://www. hermannstaedter.ro/2019/10/nachrichten-315/; 11.12.2019.

https://www.degruyter.com/page/open-access; 4.5.2020. https://www.degruyter.com/?language $=$ de; 4.5.2020. https://content.sciendo.com/view/journals/gb/gb-overview. $\mathrm{xml}$; 4.5.2020.

http://rumaenien.ahk.de/fileadmin/ahk_rumaenien/Publicatii/

Presse/2017/Germania.Informatii_generale.pdf; 11.9.2019. http://www.dsksbw.de/; 4.5.2020.

http://www.uni-regensburg.de/forschung/dimos/veranstaltungen/index.html; 4.5.2020. 